\title{
Markers and metabolites related to inflammation in bronchiectasis with airflow limitation vs. chronic obstructive pulmonary disease.
}

\author{
Jee Youn Oh ${ }^{1 \#, ~ A d n a n ~ K h a n ~}{ }^{2 \#, ~ Y o u n g ~ S e o k ~ L e e ', ~ K y u n g ~ H o o n ~ M i n ~}{ }^{1}$, Gyu Young Hur ${ }^{1}$, Sung Yong \\ Lee $^{1}$, Kyung Ho Kang1, Jae Kwan Kim², Jae Jeong Shim ${ }^{1 *}$, Youngja H Park ${ }^{2 *}$ \\ ${ }^{1}$ Division of Pulmonary, Allergy and Critical Care Medicine, Department of Internal Medicine, Korea University Guro \\ Hospital, Seoul, Republic of Korea \\ ${ }^{2}$ Metabolomics Laboratory, College of Pharmacy, Korea University, Sejong, Republic of Korea \\ \#These authors contributed equally to this work
}

\begin{abstract}
Chronic obstructive pulmonary disease (COPD) is a systemic progressive inflammatory disease resulting from an abnormal inflammatory response to noxious stimuli. Bronchiectasis is also a chronic inflammatory condition, usually caused by repetitive infections. However, it is not clear whether the inflammatory signaling pathways involved are the same in both diseases. Therefore, we here compared the markers and metabolites related to inflammation in COPD and in bronchiectasis to elucidate the pathological mechanisms. We compared markers and metabolites in patients with COPD and in those with bronchiectasis with airflow limitation. The levels of the following inflammatory biomarkers were assessed: erythrocyte sedimentation rate (ESR) and C-reactive protein (CRP), interleukin (IL)-6, and club cell secretory protein (CC)-16. Additionally, high-resolution metabolomic evaluation was conducted to evaluate the differentially expressed metabolic signature of the two diseases. The ESR and CRP levels were significantly higher, and the levels of CC-16 and IL-6 tended to be higher, among patients with bronchiectasis than among those with COPD. In the metabolomics analysis, serum metabolites of biliverdin IX alpha (m/z: $\left.565.24\left[\mathrm{M}+\mathrm{H}-\mathrm{H}_{2} \mathrm{O}\right]+\right)$ and L-carnitine $(\mathrm{m} / \mathrm{z}: 184.09[\mathrm{M}+\mathrm{Na}]+)$ were significantly upregulated in sera of bronchiectasis patients. In sera of COPD patients, the levels of nicotine $(\mathrm{m} / \mathrm{z}: 163.12[\mathrm{M}+\mathrm{H}]+)$ and $\mathrm{N}$-acetyl serotonin $\left(\mathrm{m} / \mathrm{z}: 201.102\left[\mathrm{M}+\mathrm{H}-\mathrm{H}_{2} \mathrm{O}\right]+\right)$ were significantly higher. Patients with bronchiectasis with airflow limitation had higher levels of inflammatory biomarkers (ESR and CRP) and markers indicating compensation for inflammatory damage (biliverdin IX alpha and L-carnitine), than did patients with COPD. Further treatments modulating the inflammatory process may be useful in patients with bronchiectasis.
\end{abstract}

Keywords: Biomarkers, Bronchiectasis, Chronic obstructive pulmonary disorder, Club cell secretory protein, Inflammation, Metabolomics.

Accepted on June 30, 2018

\section{Introduction}

Chronic airway inflammation is the pathophysiologic hallmark of both chronic obstructive pulmonary disease (COPD) and bronchiectasis. COPD is associated with airflow limitation, which often results from an abnormal inflammatory response to noxious stimuli, such as smoking [1]. Bronchiectasis, which also results in chronic inflammation and airflow limitation, often develops secondary to repeated infections [2,3]. Activated inflammatory cells and pro-inflammatory cytokines, such as C-reactive protein (CRP) and interleukin (IL)-6, lead to systemic inflammation, which is associated with mortality and COPD comorbidities [4-7]. Moreover, club cell secretory protein-16 (CC-16) serves as an important protective and antiinflammatory mediator in the lung, and patients deficient in
CC-16 have been known to develop COPD [8,9]. However, little is known about inflammatory markers and systemic inflammation in bronchiectasis, and no previous studies have compared the markers related to inflammation in patients with COPD and those with bronchiectasis. Although these diseases share clinical features, including inflamed and easily collapsible airways, leading to frequent hospitalization, these diseases have different pathophysiology. Defining the inflammatory markers in COPD and in bronchiectasis patients may provide an opportunity to understand the different pathologic mechanisms of systemic inflammation. Highresolution metabolomics is a promising tool that can provide insight by answering a biological question and that can document the affected end-products of cellular processes that vary according to the pathological state $[10,11]$. Adding the 
power of metabolomics to research provides an unparalleled opportunity to decipher the molecular mechanisms and potential signaling pathways that involve the inflammatory factors of disease. This study aimed to investigate the differences in the markers and metabolites related to inflammation in patients with COPD and those having bronchiectasis with airflow limitation.

\section{Materials and Methods}

\section{Subjects}

Patients with bronchiectasis with airflow limitation as well as patients with COPD were enrolled in this study. These conditions were diagnosed and patients were followed-up at Korea University Guro Hospital between 2015 and 2016. Our institutional review board (\#KUGH 13246) approved the study protocol. The patients provided written informed consent, and the study conformed to the tenets of the Declaration of Helsinki. The diagnosis of bronchiectasis with airflow limitation was made according to the following criteria: 1) bronchiectasis affecting two or more lung lobes identified on computed tomography; 2) mucopurulent or purulent sputum in the stable disease state; 3) post-bronchodilator spirometryconfirmed airflow limitation, with forced expiratory volume in 1 second (FEV1)/forced vital capacity (FVC) $<70 \%$, without a history of other airway diseases; 4) no evidence of active tuberculosis or pneumonia; and 5) age over 19 years. COPD was diagnosed on the basis of the American Thoracic Society and Global Initiative for Chronic Obstructive Lung Disease guidelines, i.e., airflow limitation confirmed by postbronchodilator spirometry, with FEV1/FVC $<70 \%$, and a history of smoking or biomass exposure. All other causes of respiratory disease that could cause airflow limitation, such as asthma, bronchiectasis, lung cancer, or interstitial lung diseases, were excluded from this study. Patients with COPDbronchiectasis overlap syndrome were also excluded. Patients in the COPD and bronchiectasis groups were matched for age and predicted FEV1\% to adjust for disease severity.

\section{Clinical parameters}

We evaluated the following clinical parameters: smoking history (packs per year and smoking status), medical history, pulmonary function test results (spirometry results, lung volume, diffusing capacity of the lung for carbon monoxide [DLCO]), plain chest radiograph, chest computed tomographic image, FEV1 decline rates, number of exacerbations per year, modified Medical Research Council scale scores, St. George Respiratory Questionnaire results, and COPD assessment test scores. FEV1 decline rate was calculated at stable status based on the change in FEV1 from baseline to 1 year after enrollment. Exacerbation was defined as sustained worsening of the patient's condition from the stable state that was beyond normal day-to-day variations, was acute in onset, and necessitated a change in the regular medication and an unscheduled hospital visit due to worsening of respiratory symptoms.

\section{Serum markers related to inflammation}

Blood tests, including CRP level and ESR measurements, were performed routinely on the day of enrollment, in an overnightfasted and medication-free state. Peripheral whole-venous blood was collected in EDTA tubes and serum was prepared by centrifugation for $10-15 \mathrm{~min}$ at $4,500 \mathrm{rpm}$ and stored at $-80^{\circ} \mathrm{C}$ until analysis. IL-6 and CC-16 concentrations were measured using ELISA kits (IL-6: R\&D Systems, Oxford, UK; CC-16: BioVendor GmbH, Heidelberg, Germany).

\section{Liquid chromatography-mass spectrometry measurements}

Liquid chromatography-mass spectrometry (LC/MS) measurements were performed as previously described [12]. In brief, $50 \mu \mathrm{l}$ aliquots of serum samples were treated with acetonitrile $(1: 4, \mathrm{v} / \mathrm{v})$ and $5 \mu \mathrm{l}$ of a mixture of three stable isotope standards ([3-Methyl-13C]-Caffeine, [Dimethyl-D6]N,N-Diethyl-M-Toluamide, and $\left.\left[13 \mathrm{C}_{5}, 15 \mathrm{~N}\right]-\mathrm{L}-\mathrm{Methionine}\right)$ $(1: 4, \mathrm{vol} / \mathrm{vol})$. Samples were analyzed in duplicate using ultraperformance liquid chromatography (Agilent Q-TOF 6550, Agilent, Santa Clara, CA, USA) with iFunnel Q-TOF mass spectrometry (Agilent Q-TOF 6550, Agilent). Analytical separation was performed using a Hypersil Gold C-18 $(100 \times$ $2.1 \mathrm{~mm}$ ) $1.9 \mu \mathrm{m}$ analytical column (Thermo Fisher Scientific, Waltham, MA, USA). The column and autosampler temperatures were maintained at $45^{\circ} \mathrm{C}$ and $10^{\circ} \mathrm{C}$, respectively. Two mobile phases, solvent A (consisting of $0.1 \%$ formic acid in water) and solvent $\mathrm{B}$ (consisting of $0.1 \%$ formic acid in $\mathrm{ACN}$ ) were used.

\section{Metabolic profiling}

Mass-to-charge ratio $(\mathrm{m} / \mathrm{z})$ of ions ranging from 50 to 1000 (resolution greater than 20,000 over 15 min LC runs, with data extraction using adaptive processing) were collected as raw spectrometric data. To obtain ion intensities, raw spectrometric data were converted to the mzXML format using two software packages, i.e., MSConvert and apLCMS. Ion intensity, $\mathrm{m} / \mathrm{z}$, and retention time are defined as $\mathrm{m} / \mathrm{z}$ features. Data were further processed with normalization, scaling, and statistical analysis using MetaboAnalyst 3.0. In MetaboAnalyst 3.0, data sets were quantile normalized, log transformed, and pareto scaled to obtain more comparable individual features. Student's t-test was used to assess the statistical significance of features, with $\mathrm{p}<0.05$ with a false discovery rate (FDR)-adjusted q-value of 0.05. Supervised partial least-squares-discriminant analysis (PLS-DA) was performed using SIMCA 14.1 (Umetrics AB, Umeå, Sweden) using unit variance (UV) scaling, to detect a significant separation shift between the two groups. The metabolites with the first principal component of variable importance in projection (VIP) value $>1.5$ and $\mathrm{p}<0.05$ were considered important for identifying differentially expressed metabolites among the groups. The relative intensity of selected metabolites with VIP $>1.5$ and $p<0.05$ was analyzed using GraphPad Prism v 5.03 (GraphPad Software, La Jolla, CA, USA). More specifically, column analysis with two-tailed (unpaired) t-tests was performed. Data are presented as means 
\pm SEM, and differences with $\mathrm{p}$ values $<0.05$ were considered statistically significant. P-values were generated with $95 \%$ confidence intervals. Box and whiskers plots were generated using Whiskers: Min to Max plot.

\section{Statistical analysis}

Clinical data were presented as median and interquartile range for continuous variables and as a percentage (number) for categorical variables. Data were compared using the MannWhitney U-test for continuous variables, and Pearson's $\chi^{2}$ test or Fisher's exact test for categorical variables. Statistical significance was defined as $\mathrm{P}<0.05$. Data were analyzed using SPSS 20.0 software (IBM, Chicago, Illinois, USA).

\section{Results}

\section{Baseline characteristics of patients with bronchiectasis and COPD}

Thirty-four participants were enrolled in the study (COPD, $\mathrm{n}=17$; bronchiectasis, $\mathrm{n}=17$ ). Baseline characteristics are described in Table 1 . Over $90 \%$ of patients with COPD were male. In contrast, only $23.5 \%$ of patients with bronchiectasis were male. Patients with bronchiectasis had no smoking history, while patients with COPD had a smoking history of 40.0 (25.0-52.8) packs per year. There were no significant differences in comorbidities between the groups. Although the FEV1 was similar between groups, the FVC, total lung capacity (TLC), and vital capacity (VC) were significantly lower in patients with bronchiectasis than in patients with COPD. The DLCO/alveolar volume (VA) was significantly higher in patients with bronchiectasis than in patients with COPD. Most patients in both groups were classified as GOLD stage $\mathrm{B}(\mathrm{COPD}=58.8 \%$; bronchiectasis $=52.9 \%)$.

Table 1. Comparison of characteristics between patients with chronic obstructive pulmonary disease and bronchiectasis.

\begin{tabular}{llll}
\hline & COPD $(\mathbf{N}=17)$ & $\begin{array}{l}\text { Bronchiectasis } \\
\mathbf{1 7})\end{array}$ & $\mathbf{( N =}$ value \\
\hline Age (years) & $72.00(63.50-76.50)$ & $64.00(56.00-74.00)$ & 0.140 \\
\hline Male $(\%)$ & $94.1 \%(16 / 17)$ & $23.5 \%(4 / 17)$ & $<0.001$ \\
\hline BMl & $22.68(20.70-24.06)$ & $23.60(20.80-25.86)$ & 0.375 \\
\hline Smoking status & & & \\
\hline Former & $58.8 \%(10 / 17)$ & $0 \%(0 / 17)$ & $<0.001$ \\
\hline Current & $41.2 \%(7 / 17)$ & $0 \%(0 / 17)$ & $<0.001$ \\
\hline Packs/year & $40.00(25.00-52.75)$ & $0.00(0.00-0.00)$ & 0.310 \\
\hline Comorbidities & & & 0.208 \\
\hline CVD & $5.9 \%(1 / 17)$ & $5.9 \%(1 / 17)$ & 0.099 \\
\hline Heart disease & $52.9 \%(9 / 17)$ & $35.3 \%(6 / 17)$ & \\
\hline Pulmonary function tests & $1.62(1.06-2.35)$ & $1.31(1.02-1.58)$ & \\
\hline FEV1 L & & & \\
\hline
\end{tabular}

\begin{tabular}{llll}
\hline FEV1\% & $68.00(42.50-77.00)$ & $56.00(45.50-71.50)$ & 0.433 \\
\hline FVC L & $3.44(2.65-3.86)$ & $2.29(1.91-2.66)$ & $<0.001$ \\
\hline FVC \% & $84.00(71.50-94.00)$ & $73.00(62.50-79.50)$ & 0.034 \\
\hline FEV1/FVC \% & $55.00(41.50-60.00)$ & $55.00(49.50-65.50)$ & 0.290 \\
\hline TLC\% & $\begin{array}{l}112.00 \\
(104.00-116.50)\end{array}$ & $99.00(86.00-104.50)$ & 0.016 \\
\hline VC\% & $93.00(80.50-106.00)$ & $71.00(66.50-85.50)$ & 0.004 \\
\hline DLCO\% & $71.00(53.50-93.50)$ & $85.00(62.00-97.50)$ & 0.610 \\
\hline
\end{tabular}

Patient-reported outcomes

\begin{tabular}{llll}
\hline mMRC & $1.00(0.00-2.00)$ & $1.00(1.00-1.00)$ & 0.803 \\
\hline CAT & $13.00(9.50-21.50)$ & $16.00(11.50-18.00)$ & 0.413 \\
\hline SGRQ & $18.43(16.02-35.44)$ & $22.67(11.95-32.63)$ & 0.760 \\
\hline
\end{tabular}

Prognosis

\begin{tabular}{llll}
\hline FEV1 decline L/year & $0.08(-0.07-0.18)$ & $0.06(0.01-0.11)$ & 0.865 \\
\hline Exacerbation /year & $0.00(0.00-1.00)$ & $0.00(0.00-1.00)$ & 0.865 \\
\hline
\end{tabular}

Data are presented as median (IQR) for continuous variables and percentage (number) for categorical variables.

COPD: Chronic Obstructive Pulmonary Disease; BMI: Body Mass Index; CVD: Cerebrovascular Disease; FEV1: Forced Expiratory Volume in 1 second; FVC: Forced Vital Capacity; TLC: Total Lung Capacity; VC: Vital Capacity; DLCO: Diffusing Capacity Of The Lungs For Carbon Monoxide; mMRC: modified Medical Research Council Scale; CAT: COPD Assessment Test; SGRQ: St. George Respiratory Questionnaire.
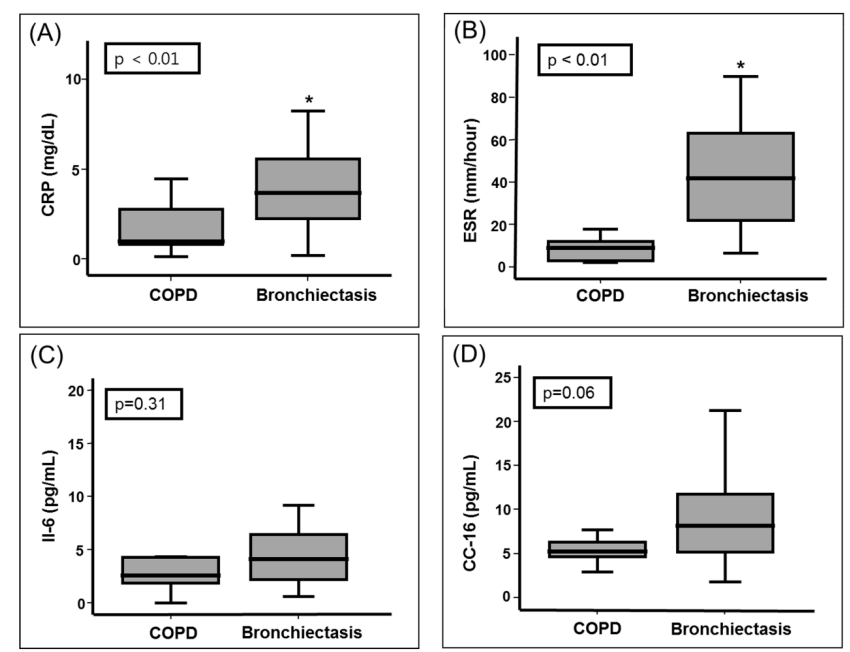

Figure 1. Comparison of serum markers related to inflammation in patients with chronic obstructive pulmonary disease (COPD) and bronchiectasis. A) Comparison of C-reactive protein (CRP) in patients with COPD and bronchiectasis; B) Comparison of erythrocyte sedimentation rate (ESR) in patients with COPD and bronchiectasis; C) Comparison of interleukin-6 (IL-6) in patients with COPD and bronchiectasis; D) Comparison of club cell secretory protein-16 (CC-16) in patients with COPD and bronchiectasis

Patient-reported scores of symptoms and quality of life were similar in both groups. There were no significant differences between groups in the FEV1 decline rates or in the number of exacerbations per year. 


\section{Comparison of serum markers related to inflammation}

CRP levels were significantly higher in patients with bronchiectasis $(3.68$ [2.06-6.81] $\mathrm{mg} / \mathrm{dl}]$ than in patients with COPD (0.99 [0.78-2.80] mg/dl, $\mathrm{P}=0.009$; Figure 1A). The ESRs were significantly higher in patients with bronchiectasis $42.00(22.00-67.95) \mathrm{mm} / \mathrm{h}$ than in patients with COPD $(9.00$ [2.50-14.50] mm/h, $\mathrm{P}<0.001$; Figure $1 \mathrm{~B})$. IL-16 levels were not significantly different between the two groups, but tended to be higher in the bronchiectasis group than in the COPD group $(\mathrm{P}=0.306$, Figure 1C). Similarly, $\mathrm{CC}-16$ levels tended to be higher in bronchiectasis patients compared to the COPD patients $(\mathrm{P}=0.057$, Figure 1D).

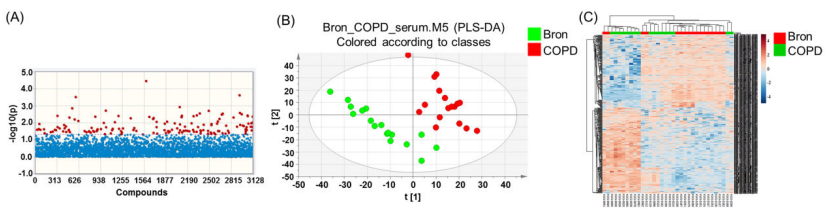

Figure 2. Differential metabolic profiles in serum of patients with chronic obstructive pulmonary disease (COPD) and bronchiectasis. A) Important features selected by the Manhattan plot with a false discovery rate (FDR)-adjusted p value threshold of 0.05 . The y-axis represents the - $\log 10$ of the raw $p$ value between compared groups, while the $x$-axis shows the compounds after normalization of their $\mathrm{m} / \mathrm{z}$ values ranging from 50 to 1000 . The dashed line represents the $F D R$ significant threshold $(q=0.05)$, which separates the significant features as pink dots from other insignificant $\mathrm{m} / \mathrm{z}$; black dots; $B$ ) Separation and classification of the metabolites by two-dimensional partial least-squares discriminant analysis (PLS-DA). In the PLS-DA score plot, each data point represents one sample. The green dots represent bronchiectasis sera and red dots represent COPD sera; C) Heat maps produced by hierarchical clustering of the most significantly different features obtained from the t-test in bronchiectasis and COPD patient groups. The green panel represents bronchiectasis sera and the red panel represent COPD sera.

\section{Differential serum metabolic profiles between COPD and bronchiectasis patients}

The raw LC-MS data were converted into the mzData format using Agilent MassHunter Qualitative Analysis software (version B.05.00; Agilent Technologies) and subsequently processed with apLCMS software using the Limma R package for quantification of ion intensities. apLCMS provided 7,481 $\mathrm{m} / \mathrm{z}$ within a range of ions set from 50 to 1000 with 20,000 resolutions. The apLCMS features produced triplicate data, which were averaged, $\log 2$ transformed, and normalized using z-transformation. To reduce the incidence of false-positives, the FDR was calculated and a Manhattan plot constructed to identify metabolites with levels that were significantly different between COPD and bronchiectasis patients. PLS-DA was then performed on the raw apLCMS data to detect a significant separation shift between comparison groups, after which hierarchical cluster analysis (HCA) was used to separate the metabolic profiles of the comparison groups by inserting raw data into MetaboAnalyst 3.0. Among 7,481 tested serum metabolites, 640 were significantly different between the COPD and bronchiectasis patient groups ( $\mathrm{q}=0.05)$ (Figure 2A).
In addition, the PLS-DA score plot significantly separated COPD patients' sera from bronchiectasis patients' sera (Figure $\left.2 \mathrm{~B}, \mathrm{R}^{2}=0.995\right)$, indicating that the serum metabolomes of the two diseases were significantly different. Furthermore, HCA using significant features obtained from the Manhattan plot showed a trend for separation between the two groups (Figure $2 \mathrm{C}$ ). These results indicated that the serum metabolome differs significantly between the COPD and bronchiectasis groups.
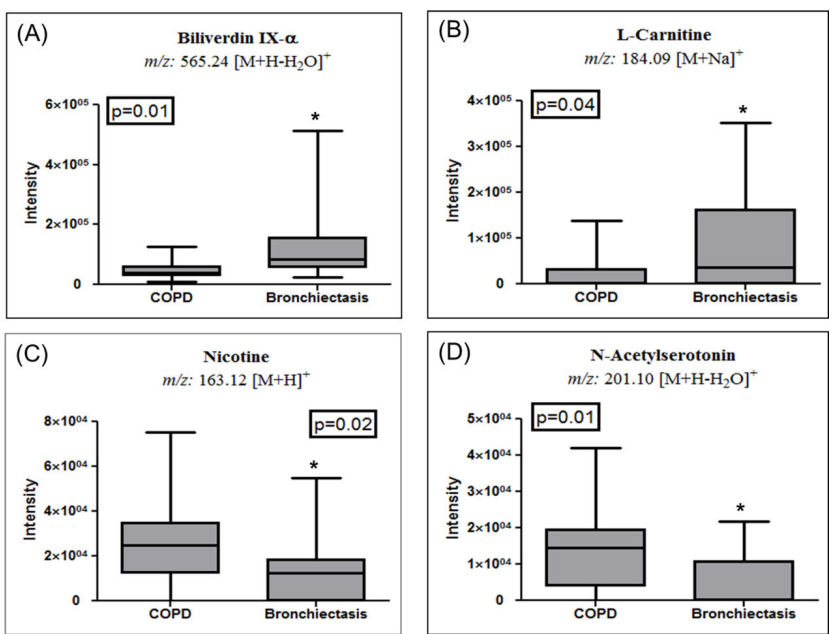

Figure 3. Relative concentrations of significant metabolite compounds in patients with chronic obstructive pulmonary disease (COPD) and bronchiectasis. A) Biliverdin IX alpha (m/z: 565.24 [M $\left.+\mathrm{H}_{-} \mathrm{H}_{2} \mathrm{O}\right]+$ ) in patients with COPD and bronchiectasis; B) $\mathrm{L}$ carnitine (m/z: $184.09[M+N a]+)$ in patients with COPD and bronchiectasis; C) Nicotine $(\mathrm{m} / \mathrm{z}$ : $163.12[\mathrm{M}+\mathrm{H}]+)$ in patients with COPD and bronchiectasis; D) $N$-acetyl serotonin (m/z: 201.102 [M $\left.+\mathrm{H}_{-} \mathrm{H}_{2} \mathrm{O}\right]+$ ) in patients with COPD and bronchiectasis.

\section{Metabolic signatures of COPD and bronchiectasis}

The significant features obtained from the Manhattan plot (FDR adjusted $\mathrm{P}<0.05$ ) were further annotated by their $\mathrm{m} / \mathrm{z}$ values using xMSannotator in order to identify the metabolites responsible for the metabolic distinctions between the groups. The following positive adducts were used $[\mathrm{M}+\mathrm{H}]+,[\mathrm{M}+\mathrm{Na}]+$, $\left[\mathrm{M}+\mathrm{NH}_{4}\right]+,\left[\mathrm{M}+\mathrm{H}-\mathrm{H}_{2} \mathrm{O}\right]+$, and $[\mathrm{M}+\mathrm{K}]+$. Based on a q value $<0.05$ and a VIP score $>2.0$, compounds related to upregulation of IL-6 and CC-16 were identified in bronchiectasis and their intensity bar graphs plotted. As shown in Figures 3A and 3B, biliverdin IX alpha (m/z: $\left.565.24\left[\mathrm{M}+\mathrm{H}-\mathrm{H}_{2} \mathrm{O}\right]+\right)$ and $\mathrm{L}$ carnitine $(\mathrm{m} / \mathrm{z}$ : $184.09[\mathrm{M}+\mathrm{Na}]+)$ were found to be significantly upregulated in bronchiectasis patients' sera. Importantly, COPD patients' sera correlated with the baseline characteristics (Table 1), but the level of nicotine (m/z: 163.12 $[\mathrm{M}+\mathrm{H}]+$ ) was significantly elevated in COPD patients as compared to bronchiectasis patients' sera (Figure 3C). In addition, as shown in Figure 3D, N-acetyl serotonin $(\mathrm{m} / \mathrm{z}$ : $\left.201.102\left[\mathrm{M}+\mathrm{H}-\mathrm{H}_{2} \mathrm{O}\right]+\right)$ was elevated in the sera of COPD patients, but not bronchiectasis patients.

\section{Discussion}

In the present study, we found that patients with bronchiectasis demonstrated higher levels of both systemic inflammatory 
biomarkers (ESR and CRP) and markers that indicate compensation for inflammatory damage (biliverdin IX alpha and L-carnitine) than did patients with COPD, which emphasizes the need to control inflammation in patients with bronchiectasis. In previous studies, patients with stable bronchiectasis had elevated levels of systemic markers of inflammation, as compared to normal control groups; some of these markers correlated with disease severity [13,14]. High levels of markers related to inflammation, similar patientrelated outcomes, and comparable lung function decline rates between patients with bronchiectasis and COPD suggest that bronchiectasis, like COPD, is a dynamic, systemic inflammatory disease.

Despite higher levels of inflammatory biomarkers (ESR and CRP), CC-16, an inflammation-responsive marker also tended to be higher in bronchiectasis patients than in COPD patients. Club cell secretory proteins are involved in airway repair after injury and detoxification of harmful substances by secreting anti-inflammatory proteins $[15,16]$. The serum level of CC-16 is reported to be lower in smokers and subjects with asthma and COPD [17-19]. However, the role of CC-16 in bronchiectasis has not been elucidated. As a consequence of the destruction of club cells, the serum concentration of CC-16 is decreased in subjects with chronic lung damage caused by tobacco smoke and other air pollutants $[8,16,20]$. In contrast, serum CC-16 levels are increase in patients with acute and chronic lung disorders characterized by an increased airway permeability [20,21]. In patients with bronchiectasis, persistent active airway inflammation caused by recurrent infections leads to angiogenesis and increased vascularity of the airways $[22,23]$. It is likely that serum CC-16 is increased in patients with bronchiectasis to compensate for inflammatory damage.

One of the important metabolomics-related findings in this study was that Biliverdin IX alpha level was significantly increased in patients with bronchiectasis. Biliverdin is a potent anti-inflammatory molecule. Biliverdin reductase $\mathrm{A}$ on the plasma membrane of macrophages rapidly converts biliverdin to bilirubin in response to inflammatory conditions. Previously, biliverdin has been shown to suppress lung permeability and lung alveolitis by suppressing serum levels of lipopolysaccharide-induced pro-inflammatory tumor necrosis factor (TNF)- $\alpha$ and IL-6 [24,25]. Contrary to these studies, our data showed a trend for an increase in IL-6 levels as well as biliverdin production in bronchiectasis patients. These discrepancies may be because biliverdin is elevated as a feedback response to compensate for inflammatory damage caused by IL-6. Similarly, L-carnitine, which has previously been shown to reduce the levels of pro-inflammatory cytokines, such as TNF- $\alpha$, IL-1 $\beta$, and IL-6 [26,27], could have been elevated in bronchiectasis patients to compensate for the levels of inflammatory cytokines. There is mounting evidence that lifelong chronic smokers have a $50 \%$ probability of developing COPD during their lifetime [28]. In accordance with a chronic smoking history [29], nicotine levels were elevated in COPD patients. Additionally, the levels of $\mathrm{N}$-acetyl serotonin, a potent antioxidant [30], may have been elevated to minimize the excessive oxidative stress caused by cigarette smoke in COPD patients [31]. This may partly explain why specific anti-inflammatory metabolites can be induced by strong inflammatory responses differently in patients who have either COPD or bronchiectasis.

In our study, we found that both inflammatory and antiinflammatory biomarkers were higher in patients with bronchiectasis than in patients with COPD. This suggests that inflammation caused by COPD is related to a reduced protective response due to the destruction of cells that would protect against this. In contrast, inflammation caused by bronchiectasis is related to activated anti-inflammatory processes that are caused by repetitive infections. Modulation of inflammation may improve disease in both diseases. In patients with bronchiectasis, reducing the inflammatory process should be the priority; in patients with COPD, the use of recombinant CC-16 should be investigated [8,32]. Moreover, we suggest that bronchiectasis should be treated with anti-inflammatory agents and bronchodilators, which are currently used for COPD treatment. Proper control of inflammation and treatment of repeated infections may improve the outcomes of patients with bronchiectasis [33,34].

These results must be considered in the context of the study limitations. First, although some of our results showed a trend, they were not statistically significant, which could potentially be explained by the small sample size. Moreover, the small sample size of both groups further limited the possibility of adjusting for confounders, making it difficult to generalize our results. Second, comparison of markers of inflammation in a healthy control group was not possible. However, it is well known that patients with stable COPD have higher levels of inflammatory markers than control individuals $[35,36]$. Thus, it could be inferred that patients with bronchiectasis have higher levels of inflammatory markers than control groups. Third, we did not evaluate the characteristics and biomarkers of bronchiectasis without airflow obstruction. However, we selected age and FEV1\% predicted-matched patients with COPD as a control group to compare and evaluate the role of inflammation in the two diseases, regardless of the presence or the severity of an airflow obstruction.

Despite these limitations, a strength of this study is that it compared inflammatory markers, including metabolites, between patients with bronchiectasis that exhibit a limitation in airflow and patients with COPD, which has not been reported previously. Further studies with a larger sample size should be performed to validate the results of this study.

\section{Conclusion}

Patients who have bronchiectasis with airflow limitation have higher levels of both systemic inflammatory biomarkers (ESR and CRP) and markers indicating compensation for inflammatory damage (biliverdin IX alpha and L-carnitine). Modulating the inflammatory process in patients with bronchiectasis could improve health outcomes. 


\section{Acknowledgment}

Financial support: Supported by Korea University Guro Hospital "KOREA RESEARCH-DRIVEN HOSPITALS" Grant and Korea Health Industry Development Institute Grant HI14C2686 by Korea University.

\section{References}

1. Barnes PJ, Celli B. Systemic manifestations and comorbidities of COPD. Eur Respir J 2009; 33: 1165-1185.

2. Hurst JR, Elborn JS, De Soyza A, Consortium B-U. COPD-bronchiectasis overlap syndrome. Eur Respir J 2015; 45: 310-323.

3. Blasi F, Chalmers JD, Aliberti S. COPD and bronchiectasis: phenotype, endotype or co-morbidity? COPD 2014; 11: 603-504.

4. Barnes PJ. Chronic obstructive pulmonary disease: effects beyond the lungs. PLoS Medicine 2010; 7: e1000220.

5. Sin DD, Man SF. Why are patients with chronic obstructive pulmonary disease at increased risk of cardiovascular diseases? The potential role of systemic inflammation in chronic obstructive pulmonary disease. Circulation 2003; 107: 1514-1519.

6. Vaguliene N, Zemaitis M, Lavinskiene S, Miliauskas S, Sakalauskas R. Local and systemic neutrophilic inflammation in patients with lung cancer and chronic obstructive pulmonary disease. BMC Immunol 2013; 14: 36.

7. Yasuda N, Gotoh K, Minatoguchi S, Asano K, Nishigaki K, Nomura M, Ohno A, Watanabe M, Sano H, Kumada H, Sawa T, Fujiwara H. An increase of soluble Fas, an inhibitor of apoptosis, associated with progression of COPD. Respir Med 1998; 92: 993-999.

8. Zhu L, Di PY, Wu R, Pinkerton KE, Chen Y. Repression of CC16 by cigarette smoke (CS) exposure. PLoS One 2015; 10: e0116159.

9. Park HY, Churg A, Wright JL, Li Y, Tam S, Man SF Tashkin D, Wise RA, Connett JE, Sin, DD. Club cell protein 16 and disease progression in chronic obstructive pulmonary disease. Am J Respir Crit Care Med 2013; 188: 1413-1419.

10. Deidda M, Piras C, Bassareo PP, Dessalvi CC, Mercuro G. Metabolomics, a promising approach to translational research in cardiology. IJC Metab \& Endocr 2015; 9: 31-38.

11. Dias DA, Koal T. Progress in metabolomics standardisation and its significance in future clinical laboratory medicine. EJIFCC 2016; 27: 331.

12. Khan A, Park H, Lee HA, Park B, Gwak HS, Lee H-R, Jee SH, Park YH. Elevated metabolites of steroidogenesis and amino acid metabolism in preadolescent female children with high urinary bisphenol A levels: A highresolution metabolomics study. Toxicol Sci 2017; 160: 371-385.
13. Wilson CB, Jones PW, O'Leary CJ, Hansell DM, Dowling RB, Cole PJ, Wilson R. Systemic markers of inflammation in stable bronchiectasis. Eur Respir J 1998; 12: 820-824.

14. Hsieh MH, Fang YF, Chen GY, Chung FT, Liu YC, Wu $\mathrm{CH}$, Chang YC, Lin HC. The role of the high-sensitivity C-reactive protein in patients with stable non-cystic fibrosis bronchiectasis. Pulm Med 2013; 2013: 795140.

15. Gamez AS, Gras D, Petit A, Knabe L, Molinari N, Vachier I, Chanez P, Bourdin A. Supplementing defect in club cell secretory protein attenuates airway inflammation in COPD. Chest 2015; 147: 1467-1476.

16. Laucho-Contreras ME, Polverino F, Gupta K, Taylor KL, Kelly E, Pinto-Plata V, Divo M, Ashfaq N, Petersen H, Stripp B, Pilon AL, Tesfaigzi Y, Celli BR, Owen C A. Protective role for club cell secretory protein-16 (CC16) in the development of COPD. Eur Respir J 2015; 45: 1544-1556.

17. Knabe L, Fort A, Chanez P, Bourdin A. Club cells and CC16: another "smoking gun"? (With potential bullets against COPD). Eur Respir J 2015; 45: 1519-1520.

18. Sonar SS, Ehmke M, Marsh LM, Dietze J, Dudda JC, Conrad ML, Renz H, Nockher WA. Clara cells drive eosinophil accumulation in allergic asthma. Eur Respir J 2012; 39: 429-438.

19. Lakind J, Holgate S, Ownby DR, Mansur A, Helms P, Pyatt D, Hays SM. A critical review of the use of Clara cell secretory protein (CC16) as a biomarker of acute or chronic pulmonary effects. Biomarkers 2007; 12: 445-467.

20. Broeckaert F, Clippe A, Knoops B, Hermans C, Bernard A. Clara cell secretory protein (CC16): features as a peripheral lung biomarker. Ann N Y Acad Sci 2000; 923: 68-77.

21. Arsalane K, Broeckaert F, Knoops B, Wiedig M, Toubeau G, Bernard A. Clara cell specific protein (CC16) expression after acute lung inflammation induced by intratracheal lipopolysaccharide administration. Am J Respir Crit Care Med 2000; 161: 1624-1630.

22. Charan N, Baile E, Pare P. Bronchial vascular congestion and angiogenesis. Eur Respir J 1997; 10: 1173-1180.

23. Cole C, Carnell S, Jiwa K, Birch J, Hester K, Ward C, Simpson JS, DE Soyza ADS. S46 Neutrophil vascular endothelial growth factor (VEGF) as a driving force for angiogenesis in bronchiectasis? Thorax 2016; 71: A28A29.

24. Sarady-Andrews JK, Liu F, Gallo D, Nakao A, Overhaus M, Ollinger R, Choi AM, Otterbein LE. Biliverdin administration protects against endotoxin-induced acute lung injury in rats. Am J Physiol Lung Cell Mol Physiol 2005; 289: L1131-L1137.

25. Bisht K, Wegiel B, Tampe J, Neubauer O, Wagner K-H, Otterbein LE, Bulmer AC. Biliverdin modulates the expression of $\mathrm{C} 5 \mathrm{aR}$ in response to endotoxin in part via mTOR signaling. Biochem Biophys Res Commun 2014; 449: 94-99. 
26. Shakeri A, Tabibi H, Hedayati M. Effects of lcarnitine supplement on serum inflammatory cytokines, Creactive protein, lipoprotein (a), and oxidative stress in hemodialysis patients with Lp (a) hyperlipoproteinemia. Hemodial Int 2010; 14: 498-504.

27. Hua X, Deng R, Zhang Z, Su Z, De-Quan L, Pflugfelder SC. L-Carnitine suppresses the production of proinflammatory cytokines by preventing the hyperosmolarity-induced oxidative stress in human corneal epithelial cells. Invest Ophthalmol Vis Sci 2014; 55: 3058 .

28. Laniado-Laborín R. Smoking and chronic obstructive pulmonary disease (COPD). Parallel epidemics of the $21 \mathrm{st}$ century. Int J Environ Res Public Health 2009; 6: 209-224.

29. Chen Q, Deeb RS, Ma Y, Staudt MR, Crystal RG, Gross SS. Serum metabolite biomarkers discriminate healthy smokers from COPD smokers. PloS One 2015; 10: e0143937.

30. Jiang J, Yu S, Jiang Z, Liang C, Yu W, Li J, Du X, Wang $\mathrm{H}$, Gao X, Wang Xin. N-acetyl-serotonin protects HepG2 cells from oxidative stress injury induced by hydrogen peroxide. Oxid Med Cell Longev 2014; 2014: 310504.

31. Rahman I. Antioxidant therapies in COPD. Int J Chron Obstruct Pulmon Dis 2006; 1: 15-29.

32. Pilon AL. Rationale for the development of recombinant human $\mathrm{CC} 10$ as a therapeutic for inflammatory and fibrotic disease. Ann N Y Acad Sci 2000; 923: 280-299.

33. Altenburg J, de Graaff CS, Stienstra Y, Sloos JH, van Haren EH, Koppers RJ, van der Werf TS and Boersma WG. Effect of azithromycin maintenance treatment on infectious exacerbations among patients with non-cystic fibrosis bronchiectasis: the BAT randomized controlled trial. JAMA 2013; 309: 1251-1259.

34. Chalmers JD, Smith MP, McHugh BJ, Doherty C, Govan JR, Hill AT. Short-and long-term antibiotic treatment reduces airway and systemic inflammation in non-cystic fibrosis bronchiectasis. Am J Respir Crit Care Med 2012; 186: 657-665.

35. Wei J, Xiong XF, Lin YH, Zheng BX, Cheng DY. Association between serum interleukin-6 concentrations and chronic obstructive pulmonary disease: a systematic review and meta-analysis. PeerJ 2015; 3: e1199.

36. Pinto-Plata VM, Müllerova H, Toso JF, Feudjo-Tepie M, Soriano JB, Vessey RS, et al. C-reactive protein in patients with COPD, control smokers and non-smokers. Thorax 2006; 61: 23-28.

\section{*Correspondence to}

Jae Jeong Shim

Division of Pulmonary, Allergy and Critical Care Medicine

Department of Internal Medicine

Korea University Guro Hospital

Republic of Korea

Youngja H Park

Metabolomics Laboratory

College of Pharmacy

Korea University

Republic of Korea 